\title{
Medicinal Value Analysis of Peony Flower
}

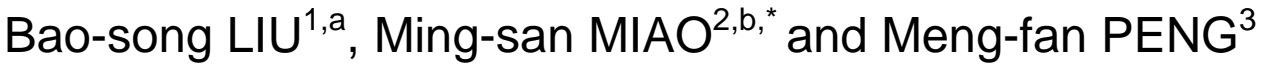 \\ ${ }^{1}$ Henan University of Chinese medicine, Zhengzhou 450046, China \\ aliubaosong647@163.com, bmiaomingsan@163.com \\ ${ }^{*}$ Corresponding author
}

Keywords: Peony flower, Chemical constituents, Pharmacological action, Therapeutic application.

\begin{abstract}
Purposes: Explore the medicinal value of peony flower. Methods: Through sorting out the literature about peony flower in CNKI, mahing a reviewed on the chemical composition, pharmacological action, therapeutic application and research direction of peony flower. Results: The Peony flower contains protein, phenolic compounds, carbohydrate, organic acids and trace elements such as zinc, iron, and copper. It has the function of nourishing blood and liver, dispersing stasis and removing stasis, and the pharmacological effects of antioxidant, anti-inflammatory, regulate the channels and relieve pain. Linoleic acid from Peony flower essential oil has the functions of anticancer, anti atherosclerosis and antihypertensive, and it can also be used as a raw material for the development of natural perfumes and essences. But in the process of peony cultivation, it is often abandoned, resulting in waste of resources. Conclusions: Peony flower has potential medicinal value and economic benefit, which deserves further study.
\end{abstract}

\section{Introduction}

Peony flower is the flower of Herbaceous perennial herbaceous plants peony, is commonly used folk diet and beautifying, there are white peony and red peony. Traditional Chinese medicine (TCM) believes that, the flower of peony tastes sweet and pungent in nature, channel tropism liver and spleen. According to the literature, the peony flowers have the functions of nourishing blood and liver, scattering depression and dispersing blood stasis, can be used for hypochon-driac pain, abdominal fullness and distention. In the folk, peony flower often be long-term consumption by the forms of porridge, cake and tea. It has the effects of nourishing blood and liver, scattering depression and dispersing blood stasis, clearing away summer heat, flatting liver and improving acuity of vision, protecting skin and beautifying. However, there are few reports about the chemical constituents, pharma-cological studies, therapeutic application and clinical application of peony flowers. The literature shows that peony flower mainly contains polyphenols, organic acids, carbohydrates, proteins and others[1], it has the pharmacological effects of anti-fibrosis of kidney, anti-inflammatory, anti-neurodegenerative, anti-cardiovascular and antioxidant [2,3]. In order to probes into its development, utilization and potential medical value, provide reference for further excavation of Peny flower, this paper making a reviewed on the main chemical components, trace elements, dietetic application and pharmacological effects of peony flower. 


\section{Chemical Components and Trace Elements}

\section{Polyphenols}

Isolation and purification of polyphenols from peony flowers by 9 different macroporous adsorption resins, a total of 9 polyphenolic compounds were detected, they were gallic acid, (+)-catechin, vanillic acid, epicatechin, p-coumaric acid, ferulic acid, rutin, Isoquercitrin and quercetin, respectively. However, protocatechuic acid, caffeic acid, lilac acid, isorhamnetin and meletin were not detected in peony flowers. After purification the content of Isoquercitrin is 2.34 times higher than that of the not purified, quercitrin is 2.03 times higher than that of the not purified [4]. Other studies have shown that the extraction rate of total polyphenols in peony flowers is related to the extraction solvent, and the extraction effect of polyphenols with 50\% ethanol is the best. The content of polyphenols in the extract from high to low was $50 \%$ ethanol > methanol acetone water $>50 \%$ methanol $>$ boiling water $>$ methanol $>$ ethanol. And the extraction efficiency of mixed solvent is better than that of single solvent [5].

\section{Carbohydrate Components}

Using the fresh petals of peony flowers as raw materials, the researchers adopted a variety of chromatographic techniques to isolate and purify their chemical constituents and to identify their structures by modern spectroscopy. A total of 5 kinds of polysaccharide compounds and one glucoside were obtained: 1,2,3,6-gallate$\beta$-D-glucose, 1,2,3,4,6-gallate- $\beta$-D-glucose, isoquercitin-3-O- $\beta$-D-(6"-gallate) glucose, Kaempferol-3-O- $\beta$-D-(6"-gallate) glucose, 1-O-galloyl- $\beta$-D-glucose and Kaempferol$3,7-\mathrm{O}-\beta-\mathrm{D}$-diglucoside. Among them, isoquercitin-3-O- $\beta-\mathrm{D}-\left(6^{\prime \prime}\right.$-gallate) glucose and Kaempferol-3-O- $\beta$-D-(6"-gallate)glucose were first isolated from the genus ${ }^{[6]}$.

\section{Volatile Components}

Peony is a traditional Chinese medicine with red peony root and white peony root. Although there are differences in the volatile components of the two petals, they are about the same mostely. The volatile constituents of red peony and white peony were mostly organic acids and alcohols, 33 kinds of volatile constituents in the petals of Wild Peony were extracted by n-hexane, and 35 kinds of volatile constituents in the petals of cultivated peony petals were extracted by n-hexane. All of them were mainly fatty acids, such as farnesol, benzyl alcohol, palmitic acid, myrtenal, camphor, $\beta$-linalool, trans orange prickly ash alcohol and others. Among them, the first two species were found in the petals of Wild Peony, and the latter two were found in the petals of cultivated Peony. These volatile compounds, benzene ethanol, farnesol, beta linalool, trans orange prickly ash alcohol are aromatic component, and phenethyl alcohol is an important and widely used edible spice [7].

\section{Trace Elements}

Modern scientific research has proved that trace elements in specific conditions are necessary to maintain the health of the body and prevent diseases. Trace elements are the basis of traditional Chinese medicine tropism and material properties, so it is great significance to study the trace elements in peony flowers. Existing studies have shown that peony flowers contains $\mathrm{Ca}, \mathrm{Mg}, \mathrm{Fe}, \mathrm{Al}, \mathrm{Zn}, \mathrm{Mn}, \mathrm{Se}$ and other trace elements, and the content decreased in turn. It is concluded that the $\mathrm{Zn} / \mathrm{Cu}$ ratio of peony flowers was well matched with $\mathrm{Zn} / \mathrm{Cu}$ in normal human serum, which is important for maintaining normal function of human body; peony flowers is rich in $\mathrm{Ca}, \mathrm{Mg}$ elements, so it can be 
used as supplement of $\mathrm{Ca}$ and $\mathrm{Mg}$ elements; The ratio of $\mathrm{Fe} / \mathrm{Mn}$ in peony flowers were higher, indicating that it has the effect of relieving the exterior and regulating the blood. In addition, the study showed that the contents of $\mathrm{Fe}$ and $\mathrm{Zn}$ were $114.25 \mathrm{mg} / \mathrm{Kg}$ and $21.45 \mathrm{mg} / \mathrm{Kg}$ respectively, and the Chinese medicine had the effect of regulating qi supplementing Qi. And the content of $\mathrm{Fe}$ and $\mathrm{Zn}$ in the flowers of Paeonia lactiflora is $112 \mathrm{mg} / \mathrm{Kg}, 23.3 \mathrm{mg} / \mathrm{Kg}$, so it can be speculated that the peony flowers also has the above effect[8].

\section{Pharmacological Effects}

\section{Anti-inflammation}

The peony flowers contains active ingredient of glycosides. Clinical studies show that total glucosides can reduce the liver toxicity of methotrexate in patients with rheumatoid arthritis; the total glucosides of paeony can be used as an additive for the treatment of such diseases [9]. In vitro studies showed that paeoniflorin pretreatment can reduce the severity of colitis and down regulated multiple inflammatory parameters such as MCP-1, C0X-2, IFN-Y, TNF-a, IL-6, and IL-17 in the colon significantly. Paeoniflorin can down regulate TLR4 expression in RAW264.7cells, inhibit the activation of NF-kB and MAPK pathway plays a protective role in the dextran sulfate induced colitis mouse model and reduces the severity of colitis [10].

\section{Antioxidant}

The extracts from peony flowers have high scavenging effects on DPPH free radicals. The scavenging rates ranged from large to small, followed by water extracts, methanol extracts, $70 \%$ methanol extracts, ethanol extracts, and $70 \%$ ethanol extracts; The extracts of Paeonia lactiflora Pall have high inhibitory ability to hydroxyl radical, and they are stronger than the concentration of $0.1 \mathrm{mg} / \mathrm{ml}$ quercetin; The inhibitory capacity of extracts from Paeonia lactiflora Pall to the superoxide anion was higher, and the methanol extract was stronger than the concentration of $0.1 \mathrm{mg} / \mathrm{ml}$ quercetin ${ }^{[11]}$. In addition, ethyl ether extract and ethyl acetate extract have strong total antioxidant capacity, DPPH free radical scavenging activity. And it can protect bovine serum albumin induced by light free radical [12].

\section{Antineoplastic}

Peony flower extract contains polyphenols, paeoniflorin and other compounds. The inhibitory effect of peony on various cancers has been proved, and the inhibition and anticancer activity on cancer cells are related to the existence of polyphenolic compounds. The total glucosides of peony can inhibit the growth of chronic myelogenous leukemia K562 cells and stop them in the G0/G1 phase; Total glucosides of paeony reduced the tumor volume and weight of transplanted K562 cells in nude mice significantly. These results suggest that total glucoside of paeony is expected to be a drug against chronic myelogenous leukemia [13]. Paeoniflorin may regulate multidrug resistance of vincristine in gastric cancer cell line SGC7901 by down regulating target genes multidrug resistance genes 1, Bal-XL and Bal-2[14].

\section{Others}

Peony flower contains paeoniflorin, albiflorin and other active ingredients, with higher $\mathrm{Fe} / \mathrm{Mn}$ values, indicating that peony flower should have the effect of relieving the exterior and regulating the blood. Studies have shown that paeoniflorin and albiflorin 
can raise the number of white blood cells in mice with blood deficiency caused by radiation therapy. They have the effect of enriching blood, and the effect of elevated white blood cells is similar [15]. Paeoniflorin can inhibit the metabolism of arachidonic acid, increase the content of 6-keto-PGF1 and decrease the TXB2 content of COX pathway products. Therefore, the neuroprotective mechanism of paeoniflorin in focal cerebral ischemia rats may be related to the improvement of the cerebral blood supply and regulation of PGI/TXA2 balance in the ischemic side [16]. Spinocerebellar ataxia is a complex autosomal heterogeneous dominant neurode- generative disorder caused by repeated encoding of polyglutamine. Peony extracts and paeoniflorin can reduce polyQ polymerization and play a role in anti neurode- generative diseases [17].

\section{Literature Status of Peony Flowers Collected in CNKI}

In the HowNet enter peony flowers, and set search keywords as "theme", the results of a total of inquiries from 2000 to August 2017, including Peony Flowers 75 articles. Only one of the 75 articles is related to clinical applications, and peony flowers appear in the form of diet therapy. Among 75 literature: A total of 12 articles on the extraction, isolation and pharmacological activities of the active constituents, accounting for $16 \%$; A total of 12 papers on pollen viability, antioxidant activity and storage life, accounting for $16 \%$; A total of 14 literatures about extraction of pigment from flower petal and extraction of volatile components, accounting for $18.6 \%$; Related to growth morphology, landscape gardening, flowering regulation, cultivation, flower bud research and other related articles 36, accounting for 48\%; Only one involving diet therapy, accounting for $1.4 \%$. Among the 12 literatures related to the extraction and pharmacological effects of active constituents, 8 studies were on antioxidant activity, accounting for $66.7 \%$; These 12 literatures all involve the extraction of polyphenols, volatile compounds and other active constituents. Details are shown in Table 1.

Table 1 Literature classification of peony flowers

\begin{tabular}{lccccc}
\hline $\begin{array}{c}\text { Literature } \\
\text { type }\end{array}$ & $\begin{array}{c}\text { pharmacology and } \\
\text { composition }\end{array}$ & pollen & petals & $\begin{array}{c}\text { clinic } \\
\text { al }\end{array}$ & othres \\
\hline Numbers & 12 & 12 & 14 & 1 & 36 \\
Percentage & $16.0 \%$ & $16.0 \%$ & $18.6 \%$ & $1.4 \%$ & $48.0 \%$ \\
\hline
\end{tabular}

Peony flower contains many kinds of the same chemical constituents as the roots of Paeonia lactiflora, that is, the pharmacodynamic material base is approximately the same as that of the root. In addition, the peony flower contains different chemical components with the peony root, and it should have its own unique pharmacological action, and it is a potential and effective drug in the clinic. However, only 12 articles related to the extraction, isolation and pharmacological activities of Paeonia lactiflora from 2000 to 2017 were collected in CNKI, only accounting for $16 \%$; there are 63 other related literatures, accounting for $84 \%$, which confirms the fact that peony flowers are more waste on the upper part of the peony.

\section{Diet Therapy Application}

The porridge of peony flower: Peony flower $6 \mathrm{~g}$, polished round-grained rice $50 \mathrm{~g}$, sugar amount. The rice cooked with the amount of water, add the peony petals cook for 2-3 
minutes, and add amount of sugar can be eaten. It has the efficacy of nourishing blood for regulating menstruation, can be used to treat liver Qi disorder, blood gas weakness, hypochondriac pain, irritability, menstrual period, abdominal pain and so on. The tea of peony flower: Peony flower amount, put into the cup, pour in boiling water, soak for a moment, then can be drink. It has the effect of nourishing blood and removing stasis, and has the preventive and therapeutic effects on freckles, chloasma, and acne and so on. The cake of peony flower: Right amount of peony petals mixed with egg white flour, fried into pancake can be eaten. It has the effect of maintain a youthful appearance and prolong life, can delay the aging of the skin.

\section{Summary}

Peony is a famous herbaceous flower. It has been cultivated for nearly 5000 years in China and ranks first among the herbs. The peony species are various, and the peony flower contains different chemical components at different times. Peony flower contains organic acids, phenols, carbohydrates, proteins, trace elements and other active and nutritional components, which are almost the same as those in Paeonia lactiflora root. Although the clinical application of the peony flower is poor, but it has similar efficacy and material basis of peony root. Therefore, it should have the pharmacological effects such as anti inflammation, ant oxidation, anti-tumor, anti neurodegeneration and blood enriching similar to the peony root. Peony flowers have been used in the folk for many years, and have been proved to have the effect of nourishing bloodand protecting liver, scattering depression and dispersing blood stasis, can improve the facial chloasma, promote metabolism, improve the immunity of the body and delay the aging of the skin.

As a non medicinal part, peony flower has potential medicinal value. But in the overall analysis, HowNet did not include the literature about the clinical application of peony flowers, which showed that Peony flowers not get full use. The peony flower related literature mostly focuses on the growth form, garden gardening, flowering regulation, cultivation and flower bud research, but not the laboratory study of medicinal value or pharmacological effect, which results in a large amount of waste of peony flowers after flowering. There is no peony flower product on the market, which leads to the peony flower, has a good role, but it cannot be widely used.

In combination with the above analysis, total glucoside of paeony is expected to be a drug for chronic myelogenous leukemia. The essential oils of peony flower are widely used in food, spices, medicine and other fields, so they can be used as a raw material for the development of natural flavors and fragrances [18]. Peony flower polyphenols and essential oils have strong antioxidant activity and can be used as a natural antioxidant in the food and pharmaceutical industry. In addition, in view of the anti oxidant and anti-inflammatory activities of peony flowers, the essential oil of flower is expected to become a new star in the cosmetics and pharmaceutical industry. To sum up, in order to promote the development of peony flower industry and guide the production and use of peony flower products in related industries, effective methods should be taken to develop medicinal preparations, daily necessities, cosmetics and health care products of peony flowers. 


\section{Acknowledgement}

This research was financially supported by the National international cooperation base ((2016)65), Zhongyuan scholars (162101510003), Henan industry university research project (172107000012).

\section{References}

[1]Zhang Li, Zhao Bingjie. Chinese journal of traditional Chinese medicine (TCM), 2016, 41(10):1835- 1842. (In Chinese)

[2]iaoYanPingl, YangJing. World latest medical information digest, 2015, 15(59):1-2.

[3]Quan Chunmei, Zhou Guangjiao, Zhu Yong. Guangzhou chemical engineering, 2016, 44 (20): 16-17+42. (In Chinese)

[4] Yu Jing, Chen Guanlin, Yang Luqi. Food research and development, 2017, 38 (07): 38-44. (In Chinese)

[5] Chen Guanlin, Liu Xuewen, Xie Yingqing. Hubei Agricultural Sciences, 20, 17, 56 (06): 1118-1122+1127. (In Chinese)

[6] Shu Xikai, Duan Wenjuan, Liu Wei. Chinese medicinal herbs, 2014, 37 (01): 6669.(In Chinese)

[7] Cheng Ming, Zhang Yongxin, Feng Xuefeng. Chinese wild plant resources, 2012, 31(03):17-19. (In Chinese)

[8] Quan Chunmei, Cao Shuai. Journal of Heihe University, 2015, 6 (03): 126-128. (In Chinese)

[9] Chen Z, Li XP, Li ZJ,et al. Reduced hepatotoxicity by total glucosides of paeony in combination treatment with leflunomide and methotrexate for patients with active rheumatoid arthritis[J], Int Immunopharmacol. 2013,15(3): 47 4-477.

[10] Zhou J, Dou W,Zhang E,et al. Paeoniflorin abrogates DSS-induced colitis via a TLR4-dependent pathway[J]. Am J Physiol Gastrointest Liver Physiol. 2014,306(1): G27-36.

[11] Jin Yingshan, Chen Manli, Jin Yinzhe. Journal of Yangzhou University (Agriculture and Life Science Edition), 2012, 33 (03): 86-90. (In Chinese)

[12] Jin YS, Xuan YH, Jin YZ,et al. Biological Activities of Herbaceous Peony Flower Extracts [J], Asian J Chem. 2013,25(7): 3835-3838.

[13] Xu HY, Chen ZW, Wu YM. Antitumor activity of total paeony glycoside against human chronic myelocytic leukemia K562 cell lines in vitro and in vivo [J]. Med Oncol, 2012, 29(2): 1137-1147.

[14] Fang S,Zhu W,Zhang Y,Shu Y,Liu P. Paeoniflorin modulates multidrug resistance of a human gastric cancer cell line via the inhibition of NF- $\kappa B$ activation[J]. Mol Med Rep, 2012, 5 (2): 351-356.

[15] Qu Shengsheng, Zhang Jianjun, Huang Yinfeng. Chinese Journal of traditional Chinese medicine, 2014, 39 (15): 2952-2955. (In Chinese)

[16] Rao Menglin, Tang Mi, He Jinyue. Chinese Journal of Pharmaceutical Sciences, 2014, 49 (01): 55-60. (In Chinese) 
[17] Chang KH, Chen WL, Lee L, et al. Aqueous Extract of Paeonia lactiflora and Paeoniflorin as Aggregation Reducers Targeting Chaperones in Cell Models of Spinocerebellar Ataxia 3[J]. Neuropharmacol. 2014, 81: 166-175.

[18] Li Hailiang, Gao Xing, Xu Fuli. Journal of Northwest Agriculture and Forestry University (NATURAL SCIENCE EDITION), 2017, 45 (05): 204-210. (In Chinese) 